EPJ Web of Conferences 53, 08012 (2013)

DOI: $10.1051 /$ epjconf/20135308012

(C) Owned by the authors, published by EDP Sciences, 2013

\title{
TARA: Forward-scattered radar detection of UHECR at the telescope array
}

\author{
J. Belz ${ }^{1, a}$, M. Abu Bakr Othman ${ }^{1}$, C. Allen ${ }^{2}$, E. Barcikowski ${ }^{1}$, D. Besson ${ }^{2}$, \\ B. Farhang-Boroujeny ${ }^{1}$, D. Ikeda ${ }^{3}$, W. Hanlon ${ }^{1}$, S. Kunwar ${ }^{2}$, J.P. Lundquist ${ }^{1}$, \\ I. Kravchenko ${ }^{4}$, S. Larson ${ }^{5}$, I. Myers ${ }^{1}$, T. Nakamura ${ }^{6}$, J.S. Rankin ${ }^{1}$, \\ H. Sagawa ${ }^{3}$, P. Sokolsky ${ }^{1}$, H. Takai ${ }^{7}$, T. Terasawa ${ }^{3}$ and G.B. Thomson ${ }^{1}$, \\ the TARA Collaboration
}

${ }^{1}$ The University of Utah, Salt Lake City, Utah, USA

${ }^{2}$ The University of Kansas, Lawrence, Kansas, USA

${ }^{3}$ Institute for Cosmic Ray Research, University of Tokyo, Kashiwa, Japan

${ }^{4}$ The University of Nebraska, Lincoln, Nebraska, USA

${ }^{5}$ Utah State University, Logan, Utah, USA

${ }^{6}$ National Institute for Polar Research, Tokyo, Japan

${ }^{7}$ Brookhaven National Laboratory, Upton, New York, USA

\begin{abstract}
Increased event statistics will be required to definitively answer the question of the origin(s) of Ultra-High Energy Cosmic Rays (UHECR). Using current technologies however, achieving the necessary statistics may be financially and practically impossible. We describe the status and plans of the TARA project, an effort to detect Ultra-High-Energy Cosmic Rays by their forward scattered or "bistatic" radar signature. Bistatic radar holds promise as a new remote sensing technique for UHECR, without the duty cycle limitations of nitrogen fluorescence detectors. Such a technique could prove key in advancing the study of UHECR beyond the constraints of the current generation of cosmic ray observatories. TARA consists of a low-VHF television transmitter illuminating the air above the Telescope Array (TA), and a set of radio receivers on the far side of TA approximately $50 \mathrm{~km}$ distant from the transmitter. We have collected radar data since April 2011 using a $2 \mathrm{~kW}$ transmitter at $54.1 \mathrm{MHz}$. Recently, we received permission to increase our broadcast power to $40 \mathrm{~kW}$ and our effective radiated power (ERP) to $6 \mathrm{MW}$. On the receiver end, we are employing software-defined radio receivers and developing real-time trigger algorithms based on the expected air shower radar echo. In addition to presenting an overview of the project status and future plans, we will present the most recent results of searches for coincidences between radar echoes and Telescope Array air shower events.
\end{abstract}

\section{INTRODUCTION}

Ultra high energy cosmic rays (UHECR) are produced in some of the most violent processes in nature. Understanding UHECR is crucial to understanding the evolution of the cosmos, as any complete model must explain not only their acceleration mechanisms, but also their propagation across cosmological distances and through the extragalactic medium.

Conventional UHECR detectors [1, 2] cover hundreds or thousands of square kilometers of the Earth's surface in order to achieve the necessary detection apertures, and are hence costly and impractical in many locations. A remote sensing technology capable of detecting cosmic-ray induced

\footnotetext{
ae-mail: belz@physics.utah.edu
}

This is an Open Access article distributed under the terms of the Creative Commons Attribution License 2.0, which permits unrestricted use, distribution, and reproduction in any medium, provided the original work is properly cited. 


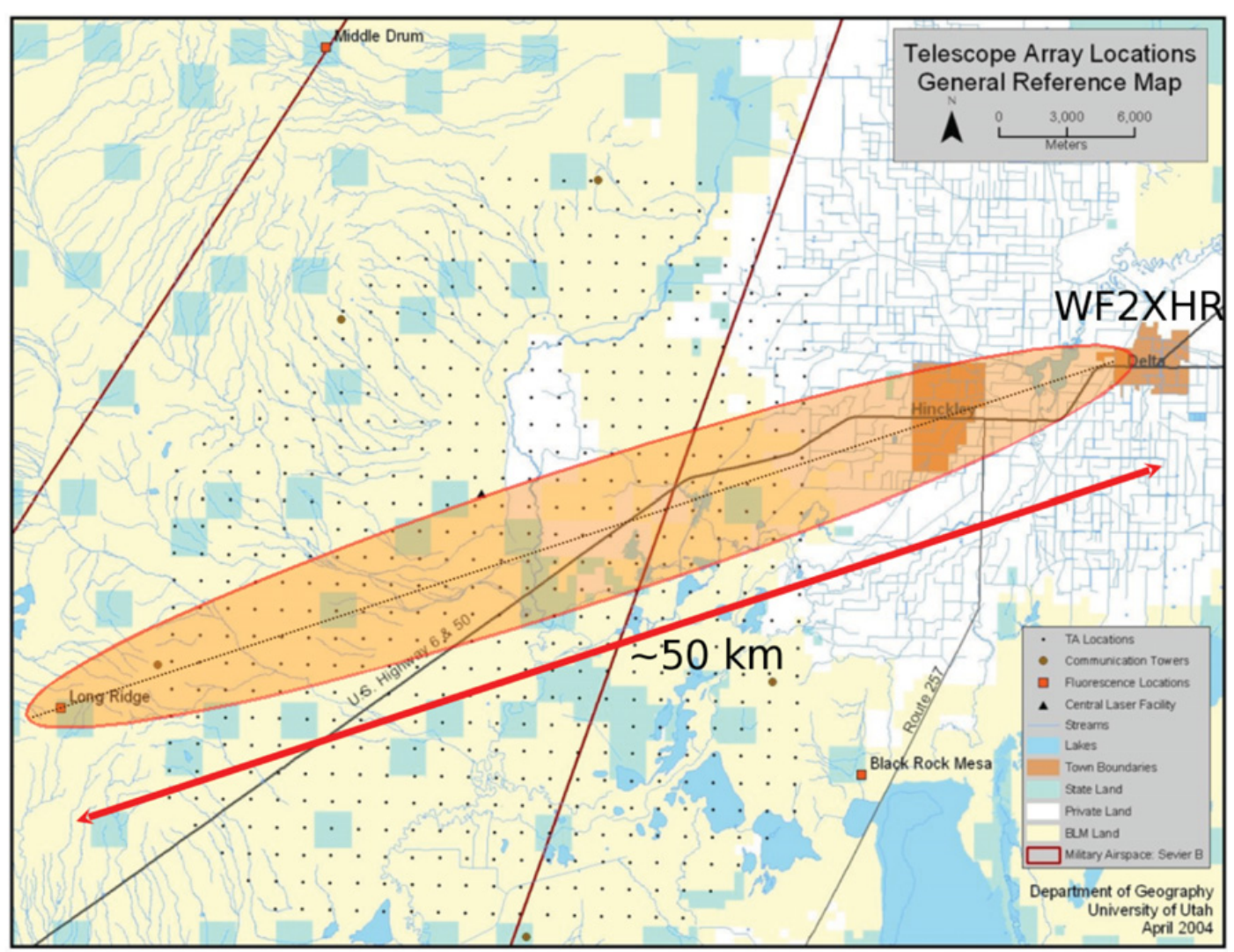

Figure 1. Map of Telescope Array showing TARA sensitive region. Transmitter located in Delta broadcasts $54.1 \mathrm{MHz}$ sine wave under FCC station license WF2XHR towards radar receiver station located at the Telescope Array "Long Ridge" fluorescence detector site.

air showers and reconstructing their astrophysically interesting parameters would be a tremendous boon to the advancement of the field. In pursuit of such a technology, The TARA (Telescope Array RAdar) project will make use of a 70 year old idea [3] and modern signal processing techniques to try to detect and eventually reconstruct air showers by their radar signatures.

\section{THE TARA CONCEPT}

TARA is the ideal configuration in which to test the radar technique. Other searches for air shower echoes have been carried out by atmospheric radar stations including Peru's Jicamarca Radio Observatory [4] and the MU Radar in Shigaraki, Japan [5]. Although anomalous signals were reported, no conventional cosmic ray detectors were on hand to confirm the signal. The first attempt to detect radar echoes in tandem with traditional surface detectors was performed by MARIACHI [6, 7], in the high radio noise environment of Long Island, New York. Again, intriguing signals were seen but uncertainties in the RF environment and limited reconstruction capabilities of the MARIACHI scintillator detectors left the results inconclusive.

As shown schematically in Figure 1, TARA is co-located with the Telescope Array (TA) detector in Millard County, Utah, U.S.A. TARA is a "bistatic" radar observatory, in which the transmitter and receiver stations are independent and remote from one another. The TARA transmitter (donated by Salt Lake City KUTV-2) broadcasts a 54.1 MHz carrier signal over the Telescope Array surface detector, 
while a receiver station located at the Long Ridge Fluorescence site searches for the forward-scattered signal. Precise GPS timing of the receiver signal allows for in-time comparison with known UHECR air shower activity in the TA surface and fluorescence detectors.

TARA is located in radio-quiet Western Utah, and the TARA transmitter is the only major source of very-high frequency (VHF) waves below the FM radio cutoff of $88 \mathrm{MHz}$. This wide-open bandwidth, in conjunction with a colocated state-of-the-art UHECR observatory make TARA the ideal test platform for the radar idea.

\section{BISTATIC RADAR PHYSICS}

Here, we briefly consider the important physics issues behind bistatic radar detection. The fundamental bistatic radar equation describes the relationship between transmitted power $P_{T}$ and received power $P_{R}[8]$ :

$$
P_{R}=P_{T} \cdot\left(\frac{G_{T}}{4 \pi R_{T}^{2}}\right) \cdot \sigma \cdot\left(\frac{G_{R}}{4 \pi R_{R}^{2}}\right) \cdot\left(\frac{\lambda^{2}}{4 \pi}\right)
$$

where $G_{T}\left(G_{R}\right)$ is the gain of the transmitting (receiving) antenna, and $R_{T}\left(R_{R}\right)$ is the distance between the transmitter (receiver) and antenna. The factor $\lambda^{2} / 4 \pi$ (where $\lambda$ is wavelength of the sounding radiation) relates the receiving antenna's gain to effective area. Each of these is under the control of the radar operator, while the factor $\sigma$ - the radar cross section RCS - is a property of the object being sounded. In the case of UHECR-induced air showers, there are large uncertainties in this factor.

The most abundant source of radar scatter comes from the ionization electrons that are produced by the energetic air shower particles as they traverse the atmosphere. These ionization electrons constitute a plasma, which can in principle reflect electromagnetic waves. Following Gorham [9], we can divide an air shower into two regions, depending on whether the ionization density exceeds the critical density at which the plasma frequency [10]

$$
\omega_{P}=\sqrt{\frac{n_{e} e^{2}}{m_{e} \epsilon_{0}}}
$$

equals the frequency of the sounding radiation.

In practice, most ionization electrons produced in a airshower will be in the underdense regime. Here, the electrons can be considered as individual agents and the RCS is simply the classical Thomson electron cross section multiplied by the number of electrons present. Unfortunately, the RF scattering that occurs in the underdense regime will be heavily suppressed by the damping that occurs due to the high rate of collisions $\left(\sim 10^{11}\right.$ per second) between the electrons and the neutral nitrogen atoms in the Earth's atmosphere [11].

It appears likely that most RF scattering will occur in the overdense part of the air shower ionization column. Here, the index of refraction becomes imaginary and reflection occurs as off of a macroscopic conductor. In Figure 2 we estimate the size of the overdense regime for a $10^{19} \mathrm{eV}$ air shower in two ways: (1) by using the Gaisser-Hillas [12] longitudinal development along with the NKG [13, 14] lateral distribution function, and (2) using a CORSIKA [15] simulation. Both calculations point to the diameter of the overdense region for $54.1 \mathrm{MHz}$ sounding radiation being of order several centimeters.

This estimate allows us to calculate two important features of the received airshower echo. First, we can estimate the received power for different transmitter/receiver arrangements. Secondly, since the transverse dimensions of the shower will be fairly small relative to the wavelength of the sounding radiation, the scattered signal will be coherent at a given longitudinal depth in the shower. A consequence of this coherence is that rays scattered from different longitudinal segments of the air shower may interfere in such a way as to produce a phase-modulated "Doppler" shifted signal. This effect has been noted in the literature previously $[16,17]$, and calculation for a typical TARA geometry is presented in 

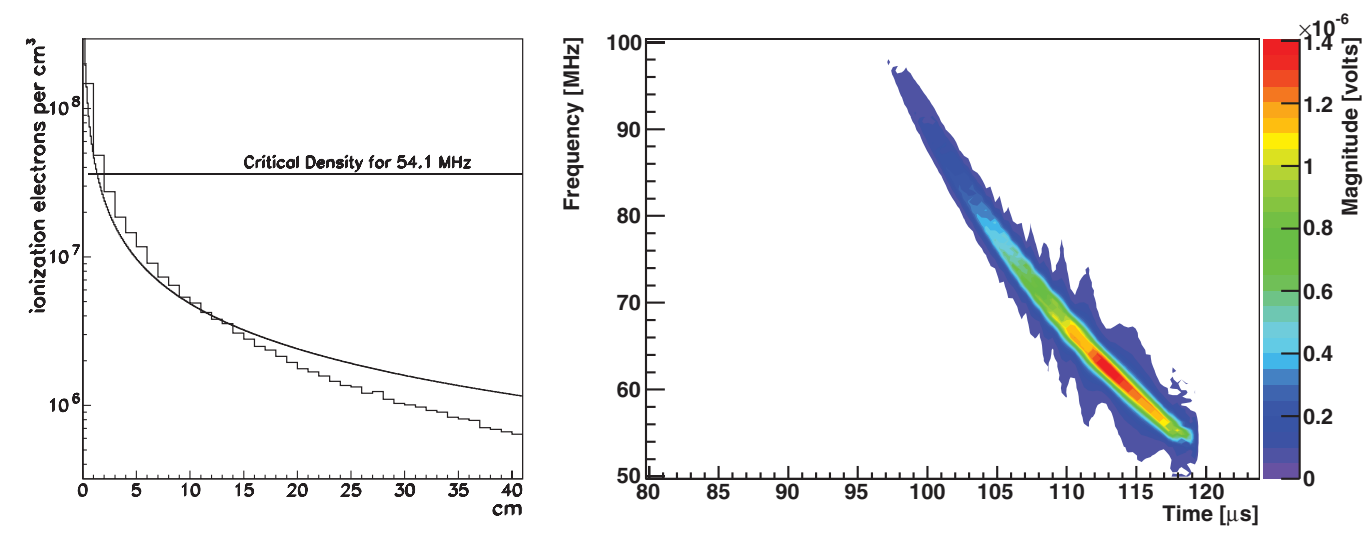

Figure 2. Left: ionization electrons per $\mathrm{cm}^{3}$ as predicted by the NKG analytical parametrization [13, 14] (curve) and CORSIKA [15] (histogram) for a vertical $10^{19} \mathrm{eV}$ air shower at ground level. Also shown is the critical density for the TARA sounding frequency of 54.1 MHz. Right: spectrogram of "chirp" for simulated air shower, initiated by $10^{19} \mathrm{eV}$ cosmic ray midway between $54.1 \mathrm{MHz}$ transmitter (TX) and receiver (RX), located $50 \mathrm{~km}$ apart. The shower is inclined at a zenith angle of $30^{\circ}$ in a plane perpendicular to a line connecting transmitter and receiver.

Figure 2. This "chirp" structure, while placing stringent requirement on the bandwidths of the receiver, also provides a unique signature by which to identify air shower echoes.

\section{TARA OBSERVATIONS TO DATE}

As of this conference, TARA has been collecting data for eight months. The transmitter is broadcasting a $54.1 \mathrm{MHz}$ sine wave at $1.5 \mathrm{~kW}$, towards the receiver station $50 \mathrm{~km}$ distant. The transmitting antenna is a single 3-element Yagi with a gain of roughly $8 \mathrm{~dB}$ over isotropic.

The receiver station consists of an array of low-VHF log periodic antennas pointed towards the transmitter. These are read out using the Ettus USRP2 software-defined radio receiver, and triggered in two ways (1) By the Telescope Array's Long Ridge fluorescence detector (FD), and read out at 12.5 Megasamples/second, and (2) with a $5 \sigma$ threshold (in sigma amplitude) self trigger, read out at 6.25 Megasamples/second.

Fluorescence trigger analysis proceeds by analyzing the waveforms of FD-triggered events for chirplike structure. As shown in Figure 3, this analysis has found no convincing structure in the most likely event candidates.

The self-triggered scheme will by definition have structure in the waveform. Here, analysis is performed by comparing the waveform's GPS timestamps with those of well-reconstructed TA surface detector events. Again, as shown in Figure 3 no statistically significant in-time signals have been observed.

\section{COMING ENHANCEMENTS}

While radar echoes have not been observed in coincidence with reconstructed Telescope Array air shower events, TARA has several enhancement projects underway which will increase both the reflected power at the receiver and the receiver triggering signal-to-noise ratio ( $\mathrm{SNR}$, in terms of received power) by large factors. These factors are summarized in Table 1 .

We will increase the transmitted power from $1.5 \mathrm{~kW}$ to $40 \mathrm{~kW}$ or more by making use of both a more powerful donated transmitter and a second transmitter which we are in the process of purchasing. In addition, we will increase the antenna gain by a factor of approximately 5 by switching our transmitting 

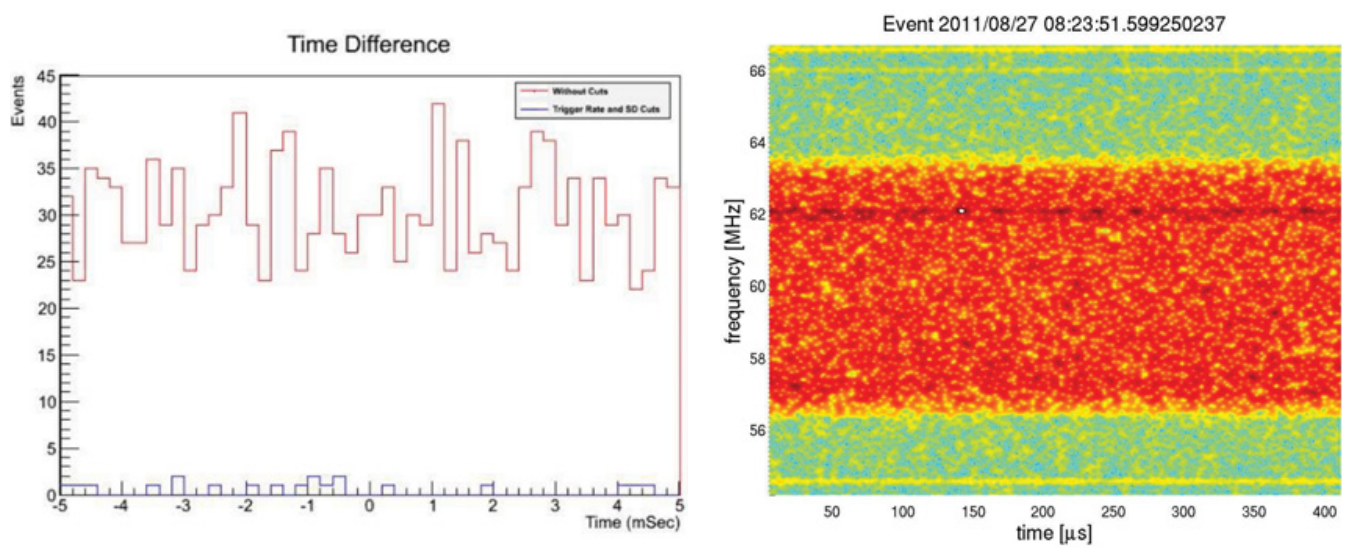

Figure 3. Left: histogram showing time difference between TARA self-triggers and TA surface detector events. The red histogram includes all surface detector triggers, and the blue histogram includes well-reconstructed UHECR air showers. Right: spectrogram (frequency versus time) for radar receiver data stream triggered by $10^{19} \mathrm{eV}$ cosmic ray air shower in the Long Ridge fluorescence detector. No chirp-like structure is observed.

Table 1. Upcoming TARA enhancement factors.

\begin{tabular}{cc}
\hline item & factor \\
\hline transmitter upgrade & 27 \\
high gain TX antenna & 5 \\
decrease detector baseline & 2.4 \\
\hline TOTAL POWER INCREASE AT RECEIVER & 324 \\
\hline \hline DECREASE RECEIVER TRIGGER SNR & 250 \\
\hline
\end{tabular}

antenna from a single 3-element Yagi to a phased array of eight 5-element Yagis. The final effective radiated power or ERP of the TARA transmitter will exceed $5 \mathrm{MW}$ continuous wave. We will gain an additional factor of 2.4 by reducing the TX $\longrightarrow$ RX baseline from $50 \mathrm{~km}$ to $40 \mathrm{~km}$. (Received power will roughly scale as baseline to the fourth power, $c f$ the bistatic radar equation Eq. (1).

At the same time, we are upgrading our radio receivers to a new system based on the National Instruments NI PXI/FlexRIO. The FlexRIO features a $250 \mathrm{MHz}$ ADC and a large-memory FPGA with complete user control. With the FlexRIO-based system we will employ a real time "matched filter" trigger algorithm to search for chirps in the $\sim 1 \mathrm{MHz} / \mu \mathrm{sec}$ range. In laboratory tests, we have achieved test signal triggering at $-18 \mathrm{~dB}$ SNR with low background using the FlexRIO system. Conservatively, we expect to do as well as $-10 \mathrm{~dB}$ SNR in the field, a factor of 250 improvement over our current $5 \sigma$ (amplitude) threshold trigger.

\section{CONCLUSIONS}

The TARA radar observatory is the most ambitious effort to date to confirm the 70 year-old idea that extensive air showers should reflect radio frequency radiation. It employs a dedicated transmitter in a radio-quiet location, colocated with the Northern Hemisphere's largest conventional cosmic ray observatory, and fast modern radio receivers in the hunt for UHECR air shower echoes. As of this conference, no coincidences between air shower-like echoes have been observed. However plans are underway to increase the reflected power at the receiver by a factor of more than 300 and to lower the receiver trigger threshold by a factor of 250 or more in SNR, thus greatly enhancing the likelihood of signal detection. 
EPJ Web of Conferences

This work is supported by the U.S. National Science Foundation grants NSF/PHY-0969865 and NSF/MRI-1126353.

We also acknowledge the generous donation of analog television transmitter equipment by Salt Lake City KUTV Channel 2.

\section{References}

[1] http://www.telescopearray.org/

[2] http://www.auger.org/

[3] P.M.S. Blackett and A.C.B. Lovell, Proc. Roy. Soc. A 177, 183 (1940)

[4] D. Wahl et al., Proc. $30^{\text {th }}$ Intl. Cosmic Ray Conf., Merida (2007)

[5] T. Terasawa et al., Proc. $31^{\text {st }}$ Intl. Cosmic Ray Conf., Lodz (2009)

[6] H. Takai, Snowbird Particle Astrophysics Conference, Snowbird, Utah (2010)

[7] http://www-mariachi.physics.sunysb.edu/

[8] N.J. Willis, Bistatic Radar, Scitech Publishing Inc. (1995)

[9] P.W. Gorham, Astropart. Phys. 15, 177 (2001)

[10] J.D. Jackson, Classical Electrodynamics, $2^{\text {nd }}$ Ed., Wiley and Sons (1975)

[11] Y. Itikawa, Phys. Fluids 16, 831 (1973)

[12] T.K. Gaisser and A.M Hillas, Proc. 15 ${ }^{\text {th }}$ ICRC, Plovdiv, Bulgaria 8, 353 (1977)

[13] K. Kamata, J. Nishimura, Suppl. Progr. Theoret. Phys 6, 93 (1958)

[14] K. Greisen, in J. G. Wilson (Editor) Prog. Cosmic Ray Physics., Vol. III, North Holland, Amsterdam, 1965, 1

[15] D. Heck et al., Forschungzentrum Karlsruhe Tech. Rep. FZKA 6019 (1998)

[16] D. Underwood, IEEE Conference Proceedings, Radar Conference (2008) http://ieeexplore.ieee.org, archive 04721089

[17] M.I. Bakunov et al., Astropart. Phys. 33, 335 (2010) 